\title{
Rôle de la nature chimique du sel utilisé pour la recherche quantitative des œufs de Parasites dans les matières fécales du porc $\left(^{*}\right)$
}

\author{
par M. BRASSINNE, M. PECHEUR et A. DEWAELE \\ (Collaboration technique: M. RENARD) \\ Chaire de Pathologie générale ( $\mathrm{P}^{\mathrm{r}} \mathrm{J}$. BROUWERS), Faculté de Médecine vétérinaire, \\ Université de Liège, Belgique
}

\begin{abstract}
Résumé
L'analyse par la méthode de Mac Master d'un même échantillon de matière fécale de porc a été répétée au moyen de 3 solutions de flottaison de même densité $(\mathrm{d}=1.200)$ mais de composition chimique différente. Le nombre d'œufs de Strongles et d'Ascaris est significativement moins élevé lorsqu'on utilise le sel $\mathrm{ZnCl}_{2}$ plutôt que $\mathrm{NaCl}$ ou $\mathrm{MgSO}_{4}$. Les auteurs signalent en outre n'avoir jamais rencontré la présence du genre Métastrongylus dans leur pays.
\end{abstract}

\section{Summary}

The analysis of the same sample of faeces of pigs was repeated by using three flotaison solutions of the same density $(\mathrm{d}=1.200)$ but of different chemical composition using the Mac Master method. The number of eggs of Strongles and Ascaris is significantly lower when $\mathrm{ZnCl}_{2}$ is used than when $\mathrm{NaCl}$ on $\mathrm{MgSO}_{4}$ is used. Besides the authors have never found the presence of genius Metastrongylus in their country.

* Travail subsidié par l'Institut pour l'encouragement de la Recherche scientifique dans l'industrie et l'Agriculture (I.R.S.I.A.), section de Recherche sur l'alimentation et les maladies du porc (CSVVZ). 


\section{Introduction.}

Nos recherches concernant l'étiologie des pneumonies si fréquemment rencontrées chez le porc, nous ont amené à définir d'abord le rôle des agents étiologiques de nature parasitaire.

Dans cette optique, nous devions en premier lieu, rechercher la présence éventuelle du genre Métastrongylus dans notre pays, vu son grand pouvoir pathogène. Pour ce faire, nous avons adopté la technique de Dunn, Gentles et White : elle permet de mettre en évidence les œufs de ce parasite chez $97 \%$ des sujets porteurs $(5,6)$. Les matières fécales sont mélangées à une solution, soit saturée $(\mathrm{d}=1,285)$ soit à $35 \%$ $(\mathrm{d}=1,204)$ de $\mathrm{Mg} \mathrm{SO}_{4}$. Ce mélange est centrifugé à 1.500 tours-minute pendant trois minutes. On recueille alors sur une lamelle les œufs qui sont remontés à la surface.

L'examen des matières fécales de 80 porcs âgés de 6 mois, ne nous a pas permis de mettre en évidence des œufs du genre métastrongylus.

Notre deuxième pôle d'intérêt a été l'étude du genre Ascaris, largement répandu chez nous, surtout parmi les porcs à l'engrais (15). On sait que la migration des larves d'Ascaris provoque des lésions pulmonaires.

Pour évaluer l'importance du rôle pathogène de ces larves dans les conditions habituelles de production, nous avons cherché à établir une éventuelle relation entre l'étendue des lésions pulmonaires à l'abattage (2) et le degré d'infestation Ascaridienne.

Devant la diversité des méthodes quantitatives utilisée par différents auteurs (4, $7,9,12,14,15)$ pour la mesure de ce degré d'infestation, nous avons retenu la méthode de Mac Master (8); elle nous a semblé la plus simple et la plus répandue ; elle a donné entière satisfaction pour les examens des matières fécales de bovins dans le service de parasitologie de notre Faculté.

La numération que nous avons entreprise a fait apparaître une grande discordance entre les chiffres selon les divers milieux de flottaison utilisées. Nous avons dès lors été amené à étudier le rôle de la nature chimique du sel sur les résultats quantitatifs obtenus pour la recherche des œufs d'Ascaris et de Strongles spp avec la méthode de numération de Mac Master.

\section{Matériel et méthodes.}

a) Echantillons.

Les échantillons de matières fécales ont été prélevés dans le rectum de porcs abattus. Chaque échantillon fut conservé à $4{ }^{\circ} \mathrm{C}$ et examiné dans les 36 heures qui ont suivi la récolte. Avant sa division, en vue des diverses méthodes d'analyses, chaque échantillon a été soigneusement homogénéisé d'abord par trituration manuelle dans un sac imperméable et souple et ensuite au moyen d'une tige en verre dans un récipient rigide.

Les trois séries d'examens relatés ci-dessous correspondent chacune à l'analyse répétée selon le même processus d'un même échantillon de matières fécales homo- 
généisé. Le premier et le deuxième échantillons (MF I et MF II), contenant des œufs d'Ascaris, ont été analysés respectivement 10 et 20 fois et le troisième (MF III), contenant des œufs de Strongles spp, a été analysé onze fois.

b) Milieu de flottaison:

Les milieux de flottaison utilisés ont été préparés par mise en solution dans de l'eau distillée de sels chimiquement pur de $\mathrm{NaCl}$, de $\mathrm{MgSO}_{4}$ ou de $\mathrm{ZnCl}_{2}$ en quantité telle que leur densité respective, mesurée à la température du laboratoire ; soient celles d'une solution saturée de $\mathrm{Na} \mathrm{Cl}(d=1.200)$.

c) Méthodes d'examens :

Toutes les manipulations et numérations ont été effectuées par la même personne.

Chaque échantillon a d'abord été divisé en autant de fractions qu'il y avait de processus d'analyse. Chacune était alors analysée trois fois suivant la même technique mais en utilisant successivement chacun des trois milieux de flottaison.

Pour la recherche des œufs d'Ascaris et de Strongles, nous avons utilisé la méthode de numération sur une lame de Mac Master. Les deux cellules de chaque lame ont été remplies au moyen de deux prélèvements successifs du mélange de matières fécales et de liquide de flottaison de façon à réduire le risque d'erreur.

d) Analyse satistique :

Le test de $t$ a été appliqué pour chaque série d'examens d'un même échantillon de façon à rechercher la signification existant entre deux séries de résultats obtenus au moyen de solutions de flottaisons différentes.

\section{Résultats :}

Les résultats obtenus peuvent être rassemblés de la façon suivante (voir tableau).

\section{Conclusion :}

Les 80 matières fécales examinées spécialement à cet effet n’ont pas révélé la présence d'œufs du genre Metastrongylus. Ce résultat est en accord avec ceux obtenus par d'autres chercheurs $(3,11,15)$ et l'on peut sans crainte de se tromper affirmer que ce parasite, bien que signalé une fois chez un sanglier (10), ne joue aucun rôle dans l'étiologie des maladies respiratoires du porc en Belgique.

Les résultats que nous publions ci-dessus, apportent la preuve de l'importance de la nature chimique du sel dans la recherche quantitative des œufs d'Ascaris et de Strongles spp dans les matières fécales de porc. Il apparaît en effet dans nos expériences que, toutes autres conditions restant par ailleurs égales, y compris la densité, l'utilisation de chlorure de zinc $\left(\mathrm{Zn} \mathrm{Cl}_{2}\right)$ dans les solutions de flottaison apporte des résultats notablement moins élevés que l'utilisation de chlorure de sodium $(\mathrm{Na} \mathrm{Cl})$ ou de sulfate de magnésie $\left(\mathrm{Mg} \mathrm{SO}_{4}\right)$. Cette conclusion est à rapprocher de celle de Dunn, Gentles et White $(5,6)$ pour qui le sulfate de magnésium $\left(\mathrm{MgSO}_{4}\right)$, de par sa nature et non par 


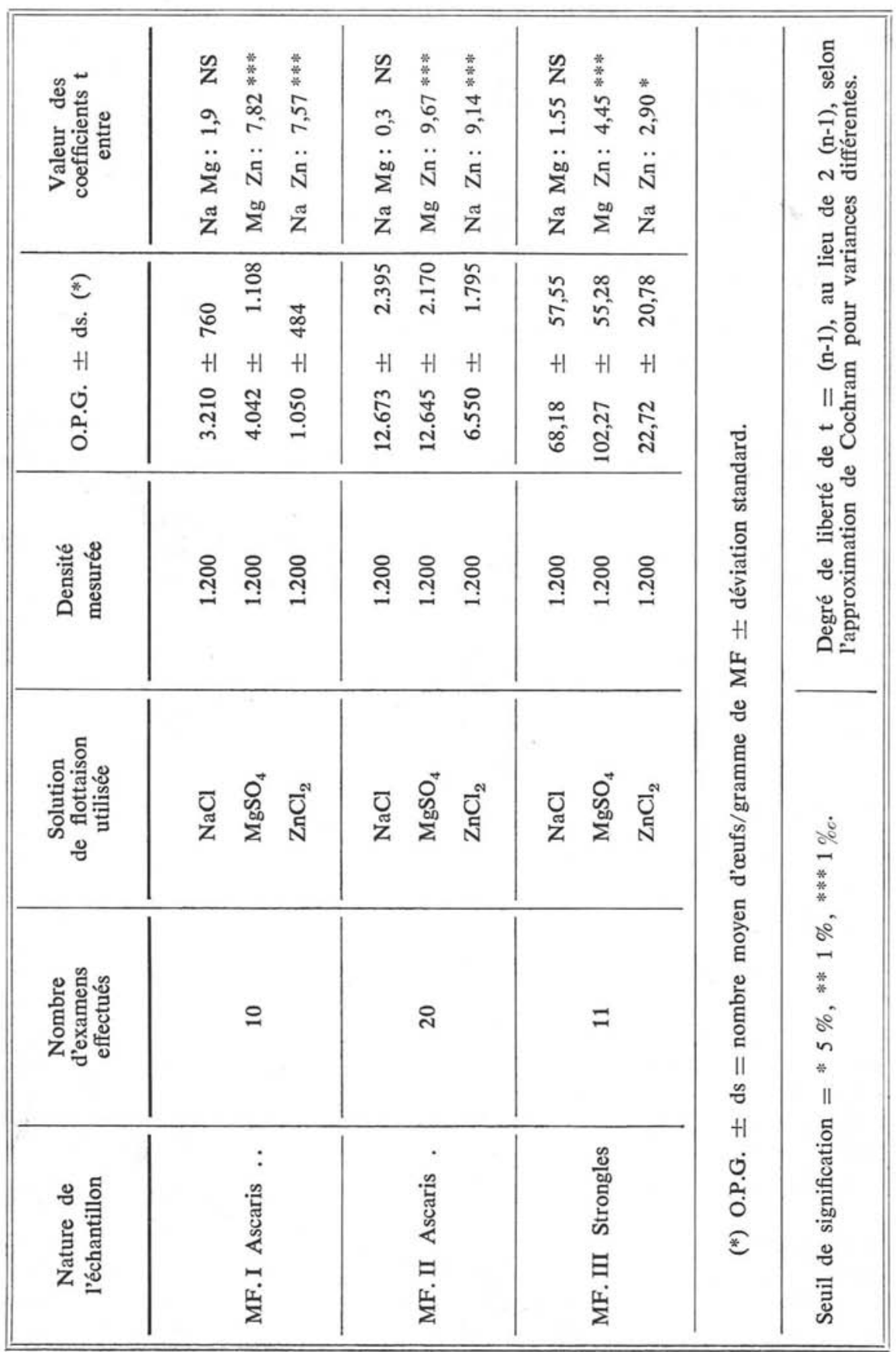


une différence de densité, convient mieux à la mise en évidence des œufs de Métastrongylus.

Toutefois, la densité de la solution paraît avoir une grande importance. En effet, l'utilisation d'une solution de chlorure de zinc $\left(\mathrm{Zn} \mathrm{Cl}_{2}\right)$ de densité supérieure nous a permis d'obtenir des résultats moyens régulièrement plus élevés. Cette observation demande cependant à être confirmée.

Notons encore que la densité d'une solution de flottaison convenable ne peut s'élever indéfiniment car, bien vite, les débris végétaux remontent également dans les couches supérieures du milieu et constituent une difficulté supplémentaire à la lecture en masquant les œufs de parasite.

En conclusion, nous pensons pouvoir, avec Bailenger (J.) et coll. (I) insister, s'il en était encore besoin, sur l'inégale valeur des méthodes de concentration parasitaire en coprologie et sur la nécessité qu'il y a de les comparer les unes aux autres si on veut soit mesurer l'intensité d'une infestation, soit diagnostiquer la totalité des cas, soit comparer des résultats obtenus dans des laboratoires différents.

\section{Bibliographie}

1. Bailenger (J.), Carcenac (F.) et Fourrier (M.-F.), 1970. - Ann. Biol. Clin.. 28, 425-430.

2. Brassine (M.), Dewaele (A.) et Brouwers (J.), 1971. - Ann. Méd. Vét., 115, 157-174.

3. Crémers (J.). - Communication personnelle.

4. Duée (J.-P.), Cornette (D.) et Moine (G.), 1969. - Rec. Med. Vet. Alfort, CXLV, 711.

5. Dunn (D.-R.), Gentles (M. A.) et White (E. G.), 1955. - Brit. Vet. J., 111, 271.

6. Dunn (D. R.) et White (E. G.), 1954. - Nature, 174, 1193.

7. Forstner (J.) et Matzke (P.). - Shell chemical Co public.

8. Kelly (W. R.), 1971. - Diagnostic clinique vétérinaire Maloine, Ed. Paris.

9. Pfeiffer (H.), 1968. - Zentbl. Vet. Med., 15 B, 895-898.

10. Pouplard (L.), 1970. - Cours de Parasitologie Vétérinaire, Bruxelles.

11. Pouplard (L.). - Communication personnelle.

12. Renault (L.), Linder (Th.), Palisse (M.) et Maire (Cl.), 1969. - Rev. Med. Vet., $120,951$.

13. TAFFs (L. F.), 1970. - Res. Vet. Sci., 11, 515.

14. Taranchon (P.), Appert (A.) et Foix (J.), 1971. - Rec. Med. Vet., 147, 369.

15. Thienpont (D.), Sierens (G.), Vanparius (O.), Lauwers (H.) et Hermans (L.), 1969. Tijdschr. Diergeneesk., 94, 1667-1678. 